\title{
Hybrid mesh for sports hernia repair
}

\author{
David S. Edelman \\ Baptist Health, Doctors Hospital, Miami, FL 33173, USA.
}

Correspondence to: Dr. David S. Edelman, Baptist Health, Doctors Hospital, 6401 SW 87th Avenue, Suite 105, Miami, FL 33173, USA. E-mail: etssurgeon@sweatstop.com

How to cite this article: Edelman DS. Hybrid mesh for sports hernia repair. Mini-invasive Surg 2017;1:31-4.

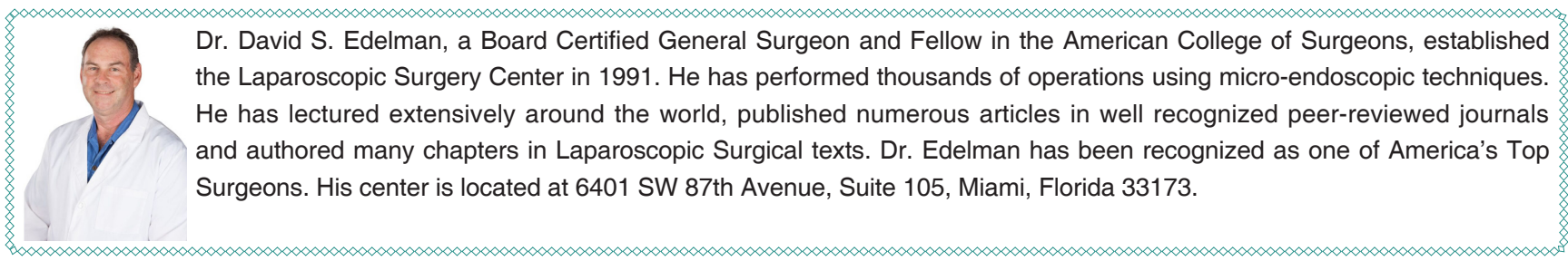

Article history:

Received: 31-10-2016

Accepted: 11-01-2017

Published: 31-03-2017

Key words:

Sports hernia,

osteitis pubis,

laparoscopic hernia repair,

inguinal hernia,

biologic mesh,

hybrid biologic mesh

\begin{abstract}
Aim: Mesh is commonly utilized in the laparoscopic repair of sports hernias. A hybrid mesh was recently released containing a single light weight layer of macroporous, polypropylene mesh between layers of biologic mesh. Having an extensive experience with laparoscopic and sports hernia repairs, a small sample of hybrid mesh was trialed. Methods: From April 2015 to August 2016, 16 male patients with sports hernias were consented for hybrid mesh repair. A prospective data base was developed and patients were followed at 1 week, 4 weeks and 4 months after surgery. Results: Ages ranged from 18 years to 43 years (average 22.9 years). Operative times ranged from $25 \mathrm{~min}$ to $75 \mathrm{~min}$ (average $42.5 \mathrm{~min}$ ). All were athletes playing basketball, soccer, baseball, football and track. There were no operative problems. Two patients developed post-operative seromas requiring radiologic drainage. All patients completed a postoperative therapy program and all have returned to their sport without problems. Conclusion: There is not one type of mesh repair that has been proven to be the most effective treatment for sports hernias. Continued follow up as well as a more structured study will be necessary to prove if hybrid mesh has long term effectiveness for the laparoscopic treatment of sports hernias. The initial study has promising findings.
\end{abstract}

\section{INTRODUCTION}

Sports hernia or Gilmore's groin was described in 1966 by Cabot and popularized in the 1980 s by Gilmore. ${ }^{[1]}$ Gilmore noted a dilated internal ring in soccer players who did not clinically have an inguinal hernia. Other pathologic findings were noted in these athletes including torn conjoined tendons, torn external oblique aponeurosis and chronic osteits pubis that did not improve with conservative treatment. It was not until 1992 that the term "sports hernia"[2] or "sportsman hernia" was introduced to define a tear in the posterior inguinal floor or transversalis fascia. Most physicians describe a lack of physical findings in the athlete's

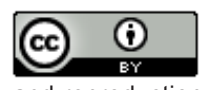$$
\text { Lice }
$$
identical terms
This is an open access article licensed under the terms of Creative Commons Attribution 4.0 International License (https://creativecommons.org/licenses/by/4.0/), which permits unrestricted use, distribution,

For reprints contact: service@oaepublish.com

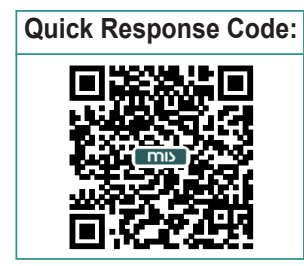


groin and were not able to demonstrate a definite inguinal hernia on exam. Surgery to repair these hernias has been popularized in the United States by Dr. William C. Meyers.

Many approaches to the treatment of this condition have been described in the literature and not one approach has been studied to be superior to any other. The laparoscopic approach to repair inguinal hernias has been demonstrated to be safe and effective. Using mesh or a synthetic prosthesis is commonplace. Despite the availability of different types of mesh, no one mesh has yet to be proven applicable to all patients or hernia repairs. Biologic mesh is designed to leave behind a minimal amount of foreign material and reduce the inflammatory response associated with polypropylene mesh. This has theoretical advantages for the athlete. Biologic mesh has been shown to be a safe and effective alternative to polypropylene mesh. ${ }^{[3]}$ However, studies on incisional hernias using biologic mesh have found late recurrences and this fact has led to incorporating an ultra-lightweight polypropylene mesh into the biologic mesh matrix. ${ }^{[4]}$ Selecting the most appropriate mesh to repair and reinforce a hernia while minimizing the failure rate but optimizing the return of the athlete to their sport is mandatory. In 2014, a hybrid mesh was released having a 6 layers of porcine small intestine sub mucosa covering a lightweight, macroporous, polypropylene mesh (Zenapro, COOK Surgical). This mesh was the basis of this study.

\section{METHODS}

Patients were seen and examined because of a suspected sports hernia. All patients had an magnetic resonance imaging (MRI) showing signs of a rectus abdominis injury or chronic osteitis pubis that persisted after a trial of conservative therapy including rest, non-steroidal anti-inflammatory medication (NSAIDs) and physical therapy. Proper informed consent was obtained on all patients. Surgery was performed on an outpatient basis. Patients were seen 7 days to 10 days after surgery and started on a rigid physical therapy program over 4 weeks. They were seen at again at 4 weeks post-op before being released to full contact. The athletes were seen for a final visit at 4 months after surgery.

A modified, double incision, total extra-peritoneal (TEP) hernia repair was performed. Patients were placed supine on the operating room table under a general endotracheal anesthesia. Five $\mathrm{mL}$ of $0.5 \%$ bupivacaine at each of the 2 skin incisions. Ten $\mathrm{mL}$ of bupivacaine was injected into the pre-peritoneal space at the completion of the procedure. A balloon cannula was used at the umbilicus to create a space for surgery down to the pubis in the pre-peritoneum. Stay sutures of 0 Vicril were placed in the fascia to hold a $12 \mathrm{~mm}$ Hasson cannula in place for the 0 degree laparoscope. Insufflation of carbon dioxide gas at a $12 \mathrm{mmHg}$ pressure was used for the surgery. A single, $5 \mathrm{~mm}$ cannula was placed in the midline, $6 \mathrm{~cm}$ below the umbilicus. A flat dissector was used at the $5 \mathrm{~mm}$ portal to dissect out the cord structures away from the pubis exposing the epigastric vessels, iliopubic tract and both inguinal areas looking for pathology. Once the dissection was complete, a $10 \mathrm{~cm} \times 15 \mathrm{~cm}$ hybrid mesh was opened on the operative field and moistened in $20 \mathrm{~mL}$ of bupivacaine prior to rolling it up and introducing it into the pre-peritoneal space. The flat dissector was used to position the mesh over the cord structures to the lateral edge of the balloon dissection and past the midline under the pubis. Four absorbable tacks were used to hold the mesh in place - superior medial, superior lateral, midline pubis and inferior into the lacunar ligament near the femoral canal. Five $\mathrm{mL}$ of fibrin sealant was then sprayed on both sides of the mesh. The remaining bupivacaine was injected into the pre-peritoneal space before removing all of the $\mathrm{CO}_{2}$ gas. The umbilical fascia was closed with a 0 Vicril suture and both skin incisions were closed with a subcuticular, 4-0 monocryl suture followed by skin glue.

\section{RESULTS}

From April 2015 to August 2016, 16 male athletes with a diagnosis of a sports hernia were consented for hybrid mesh repair. Their ages ranged from 18 years to 43 years with an average age of 22.9 years. Operative times ranged from 25 min to 75 min with an average of $42.5 \mathrm{~min}$. The athletes played sports including: soccer (5), basketball (3), track (3), football (2), baseball (1), weight lifting (1) and ultimate frisbee (1). There were no operative complications. Two patients (soccer) developed seromas overlying the urinary bladder causing intense pressure. Interventional radiology was consulted for drainage of these sterile fluid collections 2 weeks after surgery. All patients completed a postoperative therapy program and all have returned to their sport without problems.

\section{DISCUSSION}

Sports hernia involves a set of injuries in the abdominal wall and pelvis causing a weakness of the posterior inguinal wall. It is a chronic, activity related groin pain that is worsened by turning or twisting movements. Athletes can usually play through the pain but by the day following the activity, there is pain in the groin on the affected side. Rest is beneficial but resumption 
of the activity causes the pain to recur. Athletes will commonly describe that pain occurs while running and then attempting to move quickly in an opposite direction. They may find it difficult to go from a stationary position and initiate a running motion.

The physical exam is frequently not helpful in the work up of groin pain, but certain subtle findings on the exam of an injured athlete are important. Adductor tightness and pain at the inferior pubic insertion is not uncommon when the adductor longus tendon is involved in the injury. Rectus abdominis injury can elicit tenderness on the anterior pubic bone but generalized osteitis pubis will also cause tenderness to palpation in that area. With the athlete standing, palpation of the posterior pubic area and posterior inguinal floor can find cause pain or alternatively, a laxity of the posterior inguinal floor is appreciated. I will have the athlete lay supine on my exam table while placing my index finger into the external ring while having the athlete do a bilateral straight leg raise while their arms are lifted to the ceiling. I find that the same laxity or pain in the inguinal floor is a reproducible physical finding on patients with a "sports hernia".

An ultrasound can demonstrate a classic inguinal hernia and is an adequate study to go forward with surgical treatment. An MRI is commonly obtained to look at the pelvis and hip for musculo-skeletal injuries that might benefit from orthopedic consultation.

Sports hernia can be managed either non-operatively or operatively. Non-operative management consists of a combination of rest, NSAIDs, corticosteroid injections or platelet derived plasma injections, all followed by physical therapy. Athletes can return to sports in 3-4 weeks if they are pain free. However, if after 6-12 weeks they are not pain-free, repeat MRI and operative intervention should be considered.

The operative management of sports hernias involve the re-inforcement of the posterior abdominal wall using suture as described by Meyers et al. ${ }^{[5]}$ or Minnich et al., ${ }^{[6]}$ which consists of modifications of the classic Bassini hernia repair. Alternatively, a laparoscopic repair as described by Paajanen et al. ${ }^{[7]}$ or Edelman and Selesnick ${ }^{[8]}$ involves mesh placed behind the inguinal floor in the pre-peritoneal space. Mesh is commonly used in the laparoscopic repair of inguinal hernias and sports hernias ${ }^{[9]}$ Fixing the mesh with absorbable tacks or fibrin sealant is encouraged. Presently, polypropylene is the most commonly used prosthetic. In 2006, the laparoscopic treatment of sports hernia using porcine submucosa, biologic mesh was published demonstrating excellent results. ${ }^{[8]}$ The ideal material, mesh or suture, for hernia repair should be inexpensive to produce, easy to use, promote host tissue ingrowth, result in a healed repair with equal strength to normal tissue over extended periods of time, provide resistance to infection, elicit little or no inflammatory response and inhibit adhesion or fistula formation. Surgisis was initially used as a graft material for arteries, veins, ligaments, dura, urinary bladders and wound coverage. It has also been shown to be effective in the repair of abdominal wall hernias. Biologic mesh, like porcine submucosa, acts as a scaffold for host tissue collagen to re-populate the injured area with excellent revascularization. ${ }^{[0]}$ However, over time, there has been a question of the long term durability and strength with biologic mesh. ${ }^{[4]}$ Hybrid mesh was released in 2014 to meet the unmet need of a predecessor mesh for optimizing hernia repair. By adding a very lightweight polypropylene mesh to a few layers of a biologic collagen matrix, it is hoped that a beneficial host response will result in an optimal repair. ${ }^{[11]}$ This initial study on a select group of patients suggests the benefits are excellent and supports continued investigation into the use of hybrid mesh for abdominal wall repair and re-inforcement.

\section{Financial support and sponsorship}

Nil.

\section{Conflicts of interest}

There are no conflicts of interest.

\section{Patient consent}

All involved patients gave their consent forms.

\section{Ethics approval}

IRB/Ethics review was not needed by my institution to review this data.

\section{REFERENCES}

1. Gilmore OJA. Gilmore's groin: ten years experience of groin disruption - a previously unsolved problem in sportsmen. Sports Med Soft Tissue Trauma 1991;3:12-4.

2. Malycha $P$, Lovell G. Inguinal surgery in athletes with chronic groin pain: the 'sportsman's' hernia. Aust NZ J Surg 1992;62:123-5.

3. Ansaloni L, Catena F, Coccolini F, Gazzotti F, D’Alessandro L, Pinna AD. Inguinal hernia repair with porcine smallintestine submucosa: 3 year follow-up results of a randomized control trial of Lichtenstein's repair with polypropylene mesh versus Surgisis Inguinal Hernia Matrix. Am J Surg 2009;198:303-12.

4. Jin J, Rosen MJ, Blatnik J, McGee MF, Williams CP, Marks J, Ponsky J. Use of acellular dermal matrix for complicated ventral hernia repair: does technique affect outcomes? J Am Coll Surg 2007;205:654-60.

5. Meyers WC, McKechnie A, Philippon MJ, Horner MA, Zoga AC, Devon ON. Experience with "sports hernia" spanning two decades. 
Ann Surg 2008;248:656-65.

6. Minnich JM, Hands JB, Muschaweck U, Brunt LM, Diduch DR. Sports hernia: diagnosis and treatment highlighting a minimal repair surgical technique. Am J Sports Med 2011;39:1341-9.

7. Paajanen H, Brinick T, Hermunen H, Airo I. Laparoscopic surgery for chronic groin pain in athletes is more effective than non-operative treatment: a randomized clinical trial with MR imaging of 60 patients with sportsman's hernia (Athleticpubalgia). Surgery 2011;150:99107.
8. Edelman D, Selesnick H. "Sports" hernia: treatment with biologic mesh (Surgisis): a preliminary study. Surg Endosc 2006;20:971-3.

9. Nam A, Brody F. Management and therapy for sports hernia. J Am Coll Surg 2008;206:154-64.

10. Edelman DS, Hodde JP. Bioactive prosthetic material for treatment of hernias. Surg Technol Int 2006;15:104-8.

11. Wolf M, Dearth CL, Ranallo CA, LoPresti ST, Carey LE, Daly KA, Brown BN, Badylak SF. Macrophage polarization in response to ECM coated polypropylene mesh. Biomaterials 2014;35:6838-49 